research

\title{
A vital region for human glycoprotein hormone trafficking revealed by an LHB mutation
}

\author{
Iulia Potorac1,*, Adolfo Rivero-Müller2,3,4,*, Ashutosh Trehan2,*, Michał Kiełbus4, \\ Krzysztof Jozwiak5, Francois Pralong6, Aicha Hafidi7, Albert Thiry, \\ Jean-Jacques Ménagé9, Ilpo Huhtaniemi'2,10, Albert Beckers ${ }^{1}$ and Adrian F Daly1 \\ 'Department of Endocrinology, Centre Hospitalier Universitaire de Liège, Université de Liège, \\ Domaine Universitaire du Sart-Tilman, Liège, Belgium \\ 2Department of Physiology, Institute of Biomedicine, University of Turku, Turku, Finland \\ 3Faculty of Natural Sciences and Technology, Åbo Akademi University, Turku, Finland \\ ${ }^{4}$ Department of Biochemistry and Molecular Biology, Medical University of Lublin, Lublin, Poland \\ 5 Laboratory of Medicinal Chemistry and Neuroengineering, Medical University of Lublin, Lublin, Poland \\ ${ }^{6}$ Service of Endocrinology, Diabetology and Metabolism, Department of Medicine, CHU Vaudois, \\ Lausanne, Switzerland \\ Department of Diabetology and Metabolic Diseases, Centre Hospitalier Universitaire Ibn Sina, Rabat, Morocco \\ ${ }^{8}$ Department of Pathology, Centre Hospitalier Universitaire de Liège, Université de Liège, \\ Domaine Universitaire du Sart-Tilman, Liège, Belgium \\ ${ }^{9}$ Endocrinology Practice, Fontainebleau, France \\ ${ }^{10}$ Department of Surgery and Cancer, Institute of Reproductive and Developmental Biology, Hammersmith Campus, \\ Imperial College London, London, UK \\ *(I Potorac, A Rivero-Müller and A Trehan contributed equally to this work)
}

Correspondence should be addressed to A Beckers Email albert.beckers@chu.ulg.ac.be

\begin{abstract}
Glycoprotein hormones are complex hormonally active macromolecules. Luteinizing hormone (LH) is essential for the postnatal development and maturation of the male gonad. Inactivating Luteinizing hormone beta $(L H B)$ gene mutations are exceptionally rare and lead to hypogonadism that is particularly severe in males. We describe a family with selective LH deficiency and hypogonadism in two brothers. DNA sequencing of $L H B$ was performed and the effects of genetic variants on hormone function and secretion were characterized by mutagenesis studies, confocal microscopy and functional assays. A 20-year-old male from a consanguineous family had pubertal delay, hypogonadism and undetectable LH. A homozygous c.118_120del (p.Lys40del) mutation was identified in the patient and his brother, who subsequently had the same phenotype. Treatment with hCG led to pubertal development, increased circulating testosterone and spermatogenesis. Experiments in HeLa cells revealed that the mutant LH is retained intracellularly and showed diffuse cytoplasmic distribution. The mutated LHB heterodimerizes with the common alpha-subunit and can activate its receptor. Deletion of flanking glutamic acid residues at positions 39 and 41 impair LH to a similar extent as deletion of Lys40. This region is functionally important across all heterodimeric glycoprotein hormones, because deletion of the corresponding residues in hCG, follicle-stimulating hormone and thyroid-stimulating hormone beta-subunits also led to intracellular hormone retention. This novel $L H B$ mutation results in hypogonadism due to intracellular sequestration of the hormone and reveals a discrete region in the protein that is crucial for normal secretion of all human glycoprotein hormones.
\end{abstract}

\author{
Key Words \\ - luteinizing hormone \\ - mutation \\ - hypogonadism \\ - glycoprotein hormone
}

(C) 2016 Society for Endocrinology Printed in Great Britain
Published by Bioscientifica Ltd.
Journal of Endocrinology

(2016) 231, 197-207 


\section{Introduction}

Luteinizing hormone (LH) belongs to the heterodimeric glycoprotein hormone family whose other members are human chorionic gonadotropin (hCG), folliclestimulating hormone (FSH) and thyroid-stimulating hormone (TSH) (Cahoreau et al. 2015). They share a common alpha-subunit (CGA) that is non-covalently bound to a hormone-specific beta-subunit. Inactivating germline mutations of beta-subunit genes have been described for all glycoprotein hormones apart from hCG, probably due to the importance of hCG for pregnancy success.

Inactivating mutations of the $\mathrm{LH}$ beta-subunit gene $(L H B)$ are exceptionally rare and have been reported as homozygous mutations in consanguineous kindreds; a compound heterozygous $L H B$ mutation was also identified (Weiss et al. 1992, Valdes-Socin et al. 2004, Lofrano-Porto et al. 2007, Achard et al. 2009, Basciani et al. 2012). Affected males have pubertal delay that is usually associated with arrested spermatogenesis and infertility. Females have a milder phenotype with pubertal development and spontaneous menarche that is followed by anovulatory oligomenorrhoea/secondary amenorrhoea. Males with LHB mutations respond to hCG treatment, that stimulates the LH-hCG receptor (LHCGR) in Leydig cells, leading to increased testosterone production, sexual maturation and improved spermatogenesis (Valdes-Socin et al. 2004, Lofrano-Porto et al. 2007, Achard et al. 2009, Valdes-Socin et al. 2009).

Study of rare inactivating mutations of glycoprotein hormone beta-subunit genes has provided important insights into their structural-functional relationships, their heterodimerization with CGA and their receptor interactions. Here, we report a $L H B$ mutation in two homozygous brothers that resulted in a deletion of a lysine at position 40 of the LHB peptide. Unlike other $L H B$ mutations, the mutant LHB was synthesized, formed heterodimers and activated the LHCGR in vitro. The deletion of lysine 40 led to marked intracellular retention of LHB subunit. Similar experimental deletions in the beta-subunits of the other glycoprotein hormones (hCG, FSH and TSH) led to an identical pattern of intracellular retention, indicating an important role for this region in intracellular handling and secretion of this important family of hormones.

\section{Methods}

\section{Mutation detection}

After obtaining informed consent, genetic and hormonal studies were undertaken in members of the kindred. Genomic DNA was extracted from blood leucocytes and a $1082 \mathrm{bp}$ amplicon containing the complete LHB was sequenced. Primer sequences with $3^{\prime}$ end mismatches in the $3^{\prime}$ primer (to differentiate LHB from the similar hCGB) are shown in the Supplementary Materials and methods (see section on supplementary data given at the end of this article). Thirty-one members of the kindred were screened specifically for the mutation found in the propositus and his pre-pubertal brother (Fig. 1C).

\section{Construction of mutant CDNA expression vectors}

We first generated the AmCyan-P2A-mCherry vector, where nAmCyan (containing a nuclear localization signal, NLS) and mCherry are separated by P2A (a 22 amino acid self-cleaving peptide) such that upon expression of this vector, nAmCyan goes to nucleus while mCherry is retained in the cytoplasm (Supplementary Fig. 1). The plasmid contains restriction sites to allow insertion of genes of interest to act as fusion proteins with mCherry (with nAmCyan as a reporter), or as splitting proteins from mCherry where nAmCyan is replaced with the gene of interest. The AmCyan-P2A-mCherry plasmid can be accessed via the Addgene repository (Addgene plasmid \# 45350, Supplementary Fig. 2).

The $L H B$ wild-type (WT)- and mutant (Lys40del)expressing plasmids were generated by cloning 500 basepair gBlocks (IDT) into the AmCyan-P2A-mCherry as a fusion protein with mCherry via Gibson assembly (NEB). This allows the expression of the $L H B$ gene to be monitored by nuclear-AmCyan, while the LHB-mCherry could be observed during biosynthesis, transport and secretion. The final vectors (AmCyan-P2A-WT-LHBmCherry and AmCyan-P2A-Lys40del-LHB-mCherry) were used for all in vitro studies. Similarly, gBlocks of WT FSH beta (FSHB), TSH beta (TSHB) and hCG beta (hCGB) were cloned as fusion proteins with mCherry. In order to determine if the signal peptide of LHB is affected by the downstream amino acids, we replaced the $L H B$ signal peptide in the LHB-Lys40del mutant for that of prolactin (PRL). This was done by inserting a gBlock coding 
A

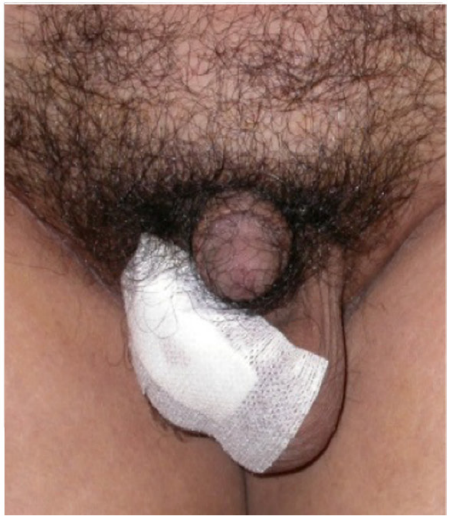

B

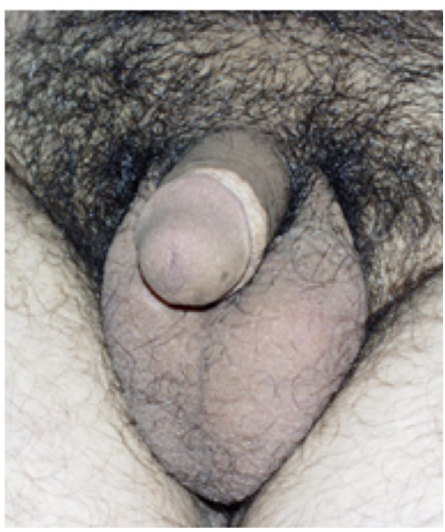

C

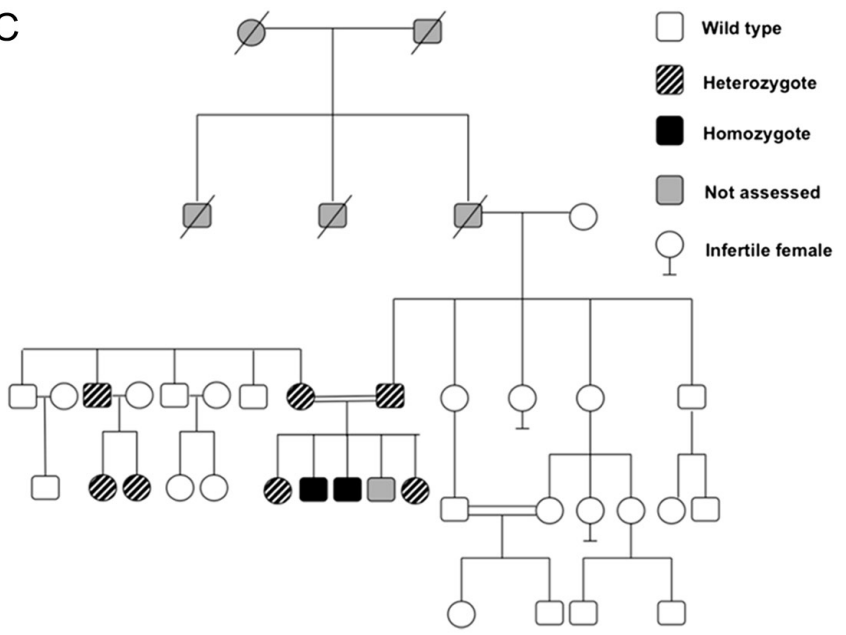

D

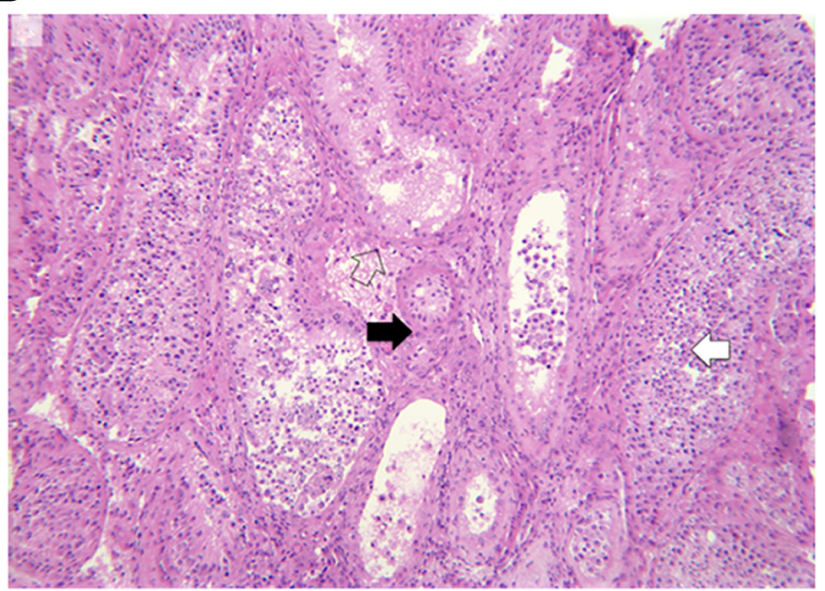

\section{Figure 1}

Clinical characterization. (A and B) External genitalia of the patient before and after treatment with hCG for 12 months is shown, with evident increase in penile length, testicular volume and pilosity. (C) Kindred diagram showing consanguinity; carriers and affected patients with the p.Lys40del mutation of $L H B$ are indicated. (D) Testicular biopsy (haematoxylin and eosin staining, 100x) showing seminiferous tubules with globally thickened basement membrane (black arrow). Spermatogenesis is present but arrested at the round spermatid stage (white arrow) and few Leydig cells are seen in the interstitial space (open arrow).

PRL-LHB-Lys40del into the AmCyan-P2A-mCherry by Gibson assembly as described above. The plasmid carrying CGA under the ubiquitin C promoter has been described previously (Ahtiainen et al. 2010).

\section{Mutagenesis}

The AmCyan-P2A-WT-LHB-mCherry, WT-FSHB, WT-hCGB or WT-TSHB plasmids were used as templates for all further mutations. All mutants were generated by REPLACR technology and were sequence-verified (Trehan et al. 2016). Primers for mutagenesis are presented in Supplementary Tables 1 and 2 . The sequences of all cloned genes are presented in Supplementary Fig. 3, and their gene products in Supplementary Fig. 4.

\section{Signal transduction studies}

HEK293 cells stably expressing a luminescent cAMP sensor (Glosensor-22F, Promega) and either LH/chorionic gonadotropin receptor (LHCGR) or follicle-stimulating hormone receptor (FSHR) have been described previously (Trehan et al. 2014) and are referred to as GS-LHCGR or GS-FSHR cells, respectively. Similar cAMP sensor cells transiently expressing the thyroid-stimulating hormone receptor (TSHR; GS-TSHR cells) were developed. HEK293 cells were transiently transfected with WT and mutant constructs of $L H B, F S H B, T S H B$ and $h C G B$, either alone or co-transfected with $C G A$, and $36 \mathrm{~h}$ later the cell culture medium was replaced with fresh DMEM/F12 medium (with $5 \%$ charcoal-treated serum) for $8 \mathrm{~h}$. The medium was then collected and stored at $-20^{\circ} \mathrm{C}$ and either

Published by Bioscientifica Ltd. 
directly used for cell stimulation or first concentrated using a microconcentrator (Millipore $10 \mathrm{kDa}$ cut-off). Activation of LHCGR, FSHR or TSHR was measured by cAMP production, as a luminescent read-out (relative light units; RLU) and is represented as area under curve. All experiments were independently repeated at least three times in triplicate.

\section{Confocal microscopy}

AmCyan and mCherry were excited with $458 \mathrm{~nm}$ and $543 \mathrm{~nm}$ laser lines, and emissions were collected at $470-579 \mathrm{~nm}$ and $578-696 \mathrm{~nm}$, respectively. Confocal microscopes (Zeiss LSM780 and LSM 510 META) were used for the experiments in living cells. Imaris software was used to export images as three-dimensional (3D) volume renders of the Z-stacks. Contrast was increased uniformly on the whole image, for WT-LHB, FSHB and hCGB to better represent the secretion vesicles that are heavily secreting their respective hormone subunits out of the cells, thus retaining very small quantities of mCherry-tagged hormones inside the cells.

\section{Measurement of LHB and LHB/CGA}

LHB and LHB/CGA were measured using commercial ELISA kits: DELFIA hLH Spec that used an immobilized antibody against hLHB and a labelled secondary antibody against a different hLHB or DELFIA hLH SPEC immobilized primary antibody with a labelled secondary antibody of DELFIA hFSH, which detects the common CGA, respectively (PerkinElmer).

\section{D modelling}

As no crystal structure for the target, LHB, is avalable, we used X-ray crystallography structure of homologous
hCG (1HRP.pdb) as a template. The residues which are not evolutionary conserved between the target and the template were replaced. All modelling and visualization was done using YASARA 11.11.2. package.

\section{Diffusion assay: fluorescence recovery after photobleaching}

Each fluorescence recovery after photobleaching experiment started with an image acquisition of a cell's cytoplasm using $561 \mathrm{~nm}$ laser $(525 / 50 \mathrm{~nm}$ excitation and 595/50 $\mathrm{nm}$ emission filter blocks) followed by a single-point bleach (non-scanning) in the cytoplasm area at full power of $405 \mathrm{~nm}$ laser for the minimum time required to photobleach most of cytoplasmic fluorescence (1-5s). Fluorescence recovery was measured by starting a time-lapse acquisition within $0.25 \mathrm{~s}$ after bleaching. Image size was $512 \times 512$ pixels and 450 scans over 120 s were performed. Pinhole size was set to the optimal value $(12.77 \mu \mathrm{m}$ confocal aperture). Normalized fluorescence was calculated as the ratio of intensity of bleached region fluorescence to intensity of reference region fluorescence. The lowest value of fluorescence intensity was estimated as 0 .

\section{Statistical analysis}

Statistical analyses were performed using GraphPad Prism 6 software using one-way ANOVA. Statistical significance and $P$ values are indicated in the respective figures.

\section{Results}

\section{Clinical studies}

A 20-year-old male of North-African descent was referred for investigation of pubertal delay. He presented

Table 1 Response to subcutaneous pulsatile GnRH pump stimulation. Samples were drawn at $12 \mathrm{~h}$ intervals from the start of the study (T0) through to $72 \mathrm{~h}$ (T72).

\begin{tabular}{|c|c|c|}
\hline & $\mathbf{T}_{\mathbf{0}}$ & $\mathbf{T}_{12}$ \\
\hline LH (RR: 2-10IU/L) & 1.1 & 0.3 \\
\hline FSH (RR: 1-8IU/L) & 31.7 & 19.8 \\
\hline$\alpha$-Subunit (RR: <0.80IU/L) & 3.70 & 2.2 \\
\hline SHBG (RR: $10-50 \mathrm{nmol} / \mathrm{L})$ & 16.8 & 15 \\
\hline Total testosterone (RR: $2.5-10 \mathrm{ng} / \mathrm{mL}$ ) & 0.77 & 0.46 \\
\hline Free testosterone (RR: $70-280 \mathrm{ng} / \mathrm{L}$ ) & 19.0 & 11.8 \\
\hline
\end{tabular}

\begin{tabular}{c}
\hline $\mathbf{T}_{\mathbf{2 4}}$ \\
\hline 0.2 \\
19.9 \\
1.7 \\
16.3 \\
0.62 \\
15.5 \\
\hline
\end{tabular}

\begin{tabular}{c}
\hline $\mathbf{T}_{\mathbf{3 6}}$ \\
\hline 0.7 \\
27.1 \\
2.5 \\
17.1 \\
0.80 \\
19.7 \\
\hline
\end{tabular}

\begin{tabular}{c}
\hline $\mathbf{T}_{\mathbf{4 8}}$ \\
\hline 0.7 \\
27.8 \\
3.89 \\
17.8 \\
1.13 \\
27.8 \\
\hline
\end{tabular}

\begin{tabular}{c}
$\mathbf{T}_{60}$ \\
\hline 0.5 \\
25.7 \\
3.40 \\
16.9 \\
0.9 \\
22.4 \\
\hline
\end{tabular}

\begin{tabular}{c}
\hline $\mathbf{T}_{\mathbf{7 2}}$ \\
\hline 0.6 \\
24.0 \\
3.69 \\
17.9 \\
1.43 \\
35.4 \\
\hline
\end{tabular}

RR, reference range; SHBG, sex hormone binding globulin.

http://joe.endocrinology-journals.org DOI: $10.1530 / \mathrm{JOE}-16-0384$
() 2016 Society for Endocrinology Printed in Great Britain 
with a hypogonadal phenotype: tall stature $(190 \mathrm{~cm})$, scant androgen-dependent pilosity, bilateral gynecomastia, micropenis and low testicular volume $(\sim 7 \mathrm{~mL})$ (Fig. 1A). He reported low libido and infrequent erections. Laboratory tests confirmed hypogonadism (total testosterone $0.64 \mathrm{ng} / \mathrm{mL}$, reference range (RR): $2.5-10 ; 2.2 \mathrm{nmol} / \mathrm{L}$, RR: 8.7-37.4) with low LH (0.4 IU/L, RR: 2-10), high FSH (19.6 IU/L, RR: 1-8) and high free $\alpha$-subunit $(3.6 \mathrm{IU} / \mathrm{L}, \mathrm{RR}$ males $<0.8)$. Inhibin B was normal (233.4 ng/L, RR: 105-439). Pituitary MRI was normal and his karyotype was 46, XY. The patient was the second of five siblings (2F, 3M) from a consanguineous kindred (Fig. 1C). One sister was investigated for infertility but had normal hormonal and ultrasound investigations; his other adult siblings had offspring. At the time of presentation, the patient's youngest brother was pre-pubertal.

A spermiogram revealed azoospermia with rare round spermatid-like cells, but no elongated spermatids. On testicular biopsy a small number of Leydig cells were seen, accompanied by relative Sertoli cell hyperplasia, and seminiferous tubules with a thickened basement membrane and arrested spermatogenesis (Fig. 1D). As the cause of hypogonadism was isolated LH deficiency, treatment with hCG (3000IU twice weekly) was started. This led to normalization of testosterone and FSH levels; after 12 months of therapy the patient had developed normal male secondary sexual characteristics with increased testicular volume $(18 \mathrm{~mL})$ (Fig. 1B). A repeat spermiogram found a marked quantitative and qualitative

A LHCGR mediated cAMP generation

Channel Overlay
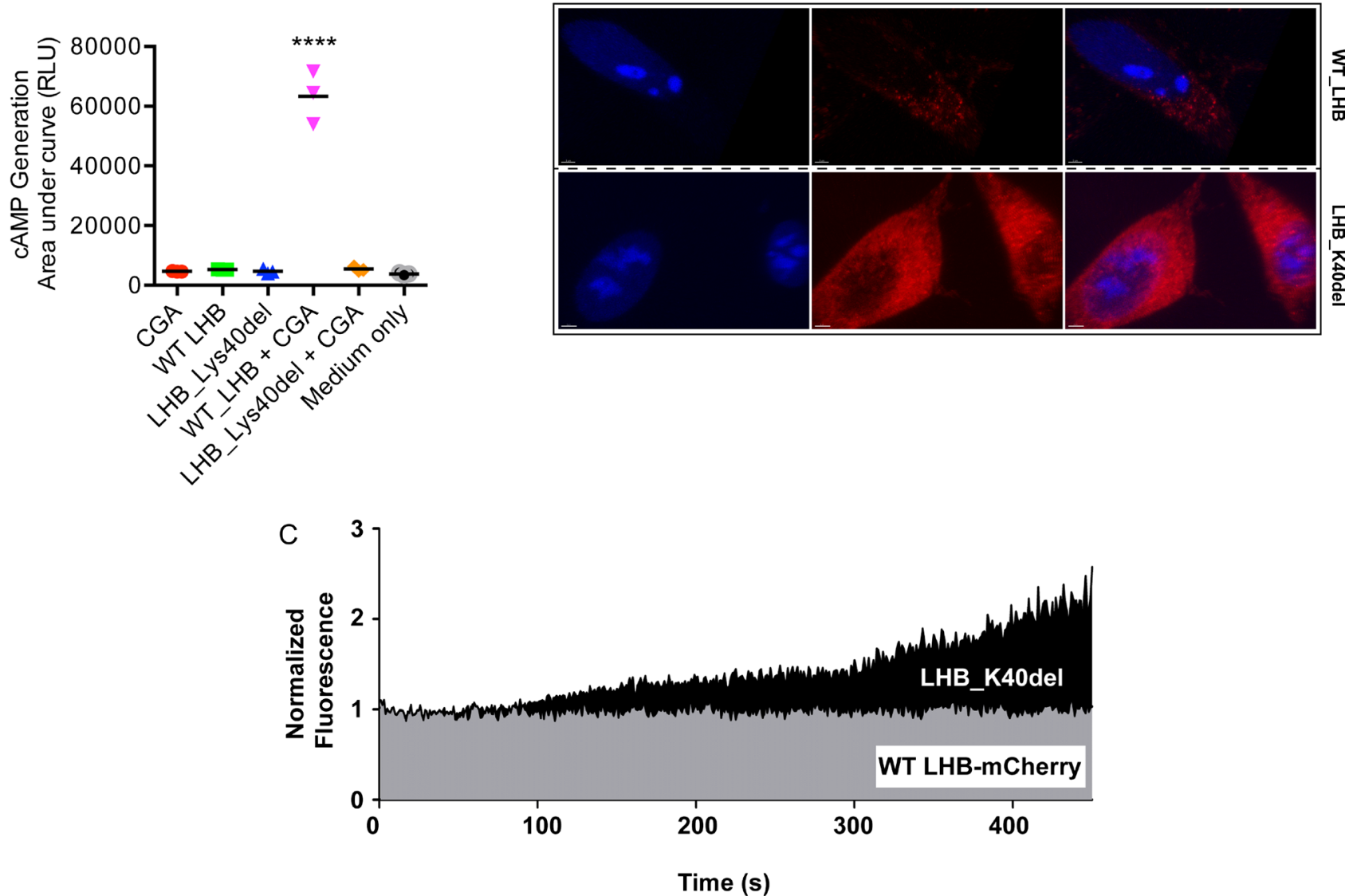

Figure 2

Functional and visual characterization of the LHB-Lys40del as compared with WT-LHB. (A) Medium was collected from HEK293 cells transfected with various plasmid combinations and used to stimulate LHCGR using GS-LHCGR reporter cells. The luminescent read-out is proportional to cAMP production. CGA alone, LHB alone or medium from untransfected cells (medium alone) resulted in no activation of the LHCGR. WT-LHB + CGA produced the expected activation, while the LHB-Lys40del +CGA mutant showed a minimal, significantly reduced response $(P$ value $<0.0001)$. (B) Confocal images of HeLa cells expressing WT-LHB shows that it is present only in secretory vesicles, while the LHB-Lys40del mutant is diffusely present throughout the cytoplasm. (C) A diffusion test shows that while the WT-LHB does not diffuse within the cell (it is strictly compartmentalized) the Lys40del freely diffuses which suggest that it is not compartmentalized but rather in the cytosol. The latter chart is representative of at least three independent experiments where up to 10 single cells were analyzed. 
improvement $(200,000 / \mathrm{mL}$ with good mobility and normal shape for $50 \%$ of spermatozoa). After 14 months of hCG therapy, treatment was discontinued for two months and a dynamic hormonal study was performed using prolonged subcutaneous pulsatile GnRH administration (gonadorelin-HRF (Tramedico); $20 \mu \mathrm{g}$ every $90 \mathrm{~min}$ for $72 \mathrm{~h}$ ) via a pump (PANOMAT-Disetronic). Circulating levels of LH, FSH, total and free testosterone, free alpha-subunit as well as sex hormone binding globulin were assessed every $12 \mathrm{~h}$ (Table 1 ). While total testosterone levels rose from $0.77 \mu \mathrm{g} / \mathrm{L}$ at $\mathrm{T}_{0}$ to $1.43 \mu \mathrm{g} / \mathrm{L}$ at $\mathrm{T}_{72} \mathrm{~h}$, both testosterone and LH remained below the lower limit of normal (Table 1).

Sequencing of the $L H B$ gene revealed a deletion of three nucleotides, c.118_120del, leading to an in-frame deletion of lysine 40 of the mature peptide (p.Lys40del). The mutation was found in a homozygous state in the propositus, his pre-pubertal brother and in a heterozygous state in his parents, his two sisters, a maternal uncle and his two daughters (Fig. 1C). During long-term follow-up of the kindred, the younger homozygous brother also displayed delayed puberty in his mid-teens that was accompanied by the same hormonal picture as his brother (no testicular biopsy was performed). Given the good clinical response to hCG treatment in other males with $L H B$ mutation-related hypogonadism, he was treated in a similar fashion to his brother and completed puberty with normal secondary sexual characteristics.

\section{Signal transduction}

As expected, neither CGA nor WT-LHB resulted in receptor activation when expressed alone in HEK293 cells. WT-LHB fused with mCherry formed heterodimers with CGA and these dimers activated the LHCGR (Fig. 2A). In contrast, co-expression of the LHB-Lys40del mutant with CGA was not able to activate LHCGR. Confocal microscopy demonstrated that WT-LHB was concentrated mainly within secretory vesicles, while the LHB-Lys40del mutant was present diffusely throughout the cell (Fig. 2B). We performed a diffusion test in which normally trafficked proteins diffuse poorly into a photo-bleached region, while non-trafficked proteins rapidly diffuse into the bleached region (Cardarelli et al. 2012). As hypothesized, the normally trafficked WT-LHB diffused poorly while the LHB-Lys40del mutant rapidly diffused to the bleached

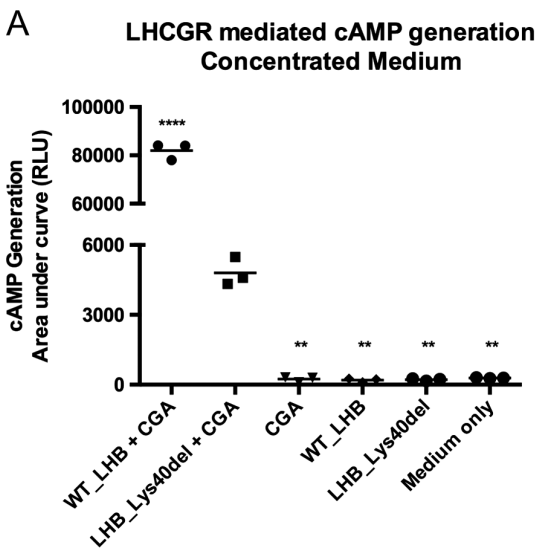

C Heterodimers/LHB

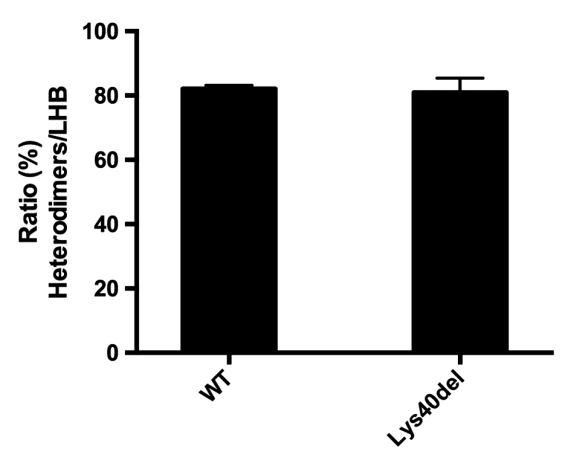

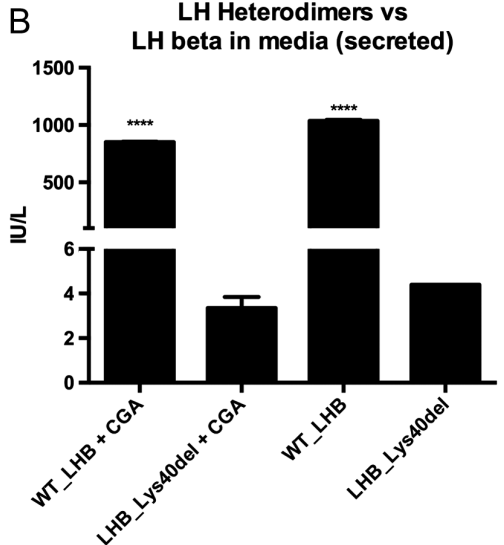

D

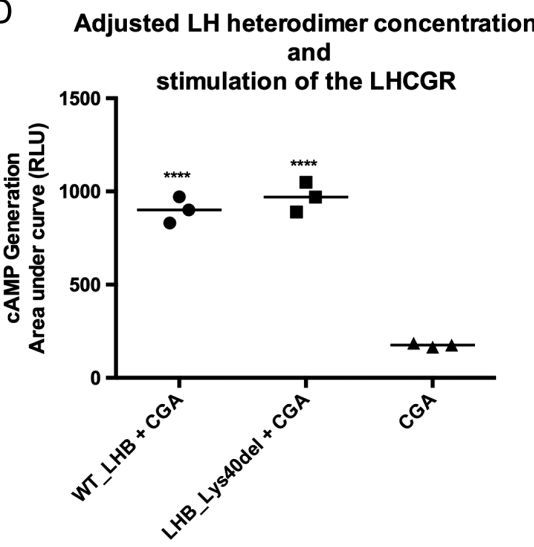

Figure 3

Heterodimerization of LHB with CGA and LHCGR activation. (A) Either WT-LHB + CGA or LHBLys40del+CGA were transiently over-expressed and media was collected $48 \mathrm{~h}$ after transfection and concentrated. The concentrated fractions were used to stimulate cells expressing the LHCGR and a CAMP sensor. The generation of CAMP was recorded. While the WT-LHB + CGA resulted in a very marked response, the LHB-Lys40del + CGA resulted in a minor response, yet significantly larger than CGA alone, LHB alone or the control media; (B) concentration of $\mathrm{LHB}$ and $\mathrm{LHB}+\mathrm{CGA}$ in the media of cells transfected with CGA and either WT-LHB or LHB-Lys40del; (C) ratio between secreted LHB and LHB + CGA heterodimers; (D) the concentration of the WT-LHB + CGA was diluted to that of the mutant (LHB-Lys40del +CGA) and then their potential to activate the LHCGR was measured as generation of CAMP, they both show identical responses demonstrating that heterodimerization to CGA and LHCGR activation are unaffected by the mutation. The statistical difference ( $P$ value $<0.0001)$ for all the comparisons is highlighted as compared with LHB-Lys40del+CGA. 
region (Fig. 2C). As nuclear-AmCyan levels were similar in WT and Lys40del-expressing cells (Fig. 2B), this indicated that the expression level of the both WT-LHB and LHB-Lys40del mutant was normal.

We then over-expressed the WT-LHB or LHBLys40del in the presence of CGA, collected the media after $48 \mathrm{~h}$ and concentrated it. Concentrated WT-LHB + CGA strongly stimulated the luminescent reporter GS-LHCGR cells but concentrated LHBLys40del + CGA showed only a small response, although it was significantly higher than CGA or WT-LHB alone, or control medium (Fig. 3A). In the concentrated media, the concentration of LHB-Lys40del was 234fold less than WT-LHB (Fig. 3B); there was no difference in the ratios of beta-subunit to heterodimer between the WT and mutated LHB (Fig. 3C). After diluting the LHB-WT 234-fold to equalize the concentration with that of LHB-Lys40del, both activated GS-LHCGR cells identically (Fig. 3D). This indicates that the LHB-Lys40del mutant heterodimerizes with CGA to a similar extent as WT-LHB and that the heterodimer, once formed, is fully functional in activating its receptor. The decreased LH seen in the clinical setting, therefore, likely is due to intracellular retention of the mutated protein.

Using 3D modelling, the Lys40 deletion appears to alter the conformation of a loop, which suggests that this region may be important for proper folding of the beta-subunit or formation of the quaternary structure (Fig. 4A and B). To determine if the conserved glutamic acid residues immediately adjacent to Lys40 contribute to biosynthesis, we generated deletions of Glu39 or Glu41. LHB-Glu39del or LHB-Glu41del resulted in the same effect as LHB-Lys40del (Fig. 4C). Mutagenesis of Lys40 to alanine, arginine or asparagine led to fully functional proteins when co-expressed with CGA that activated LHCGR to a similar extent as WT-LHB (Fig. 4C). We also ruled out that the Lys40 deletion affected secretion of LH by impairing the LHB signal peptide. We replaced the LHB signal peptide with that of prolactin (PRL-Lys40del), as the PRL signal peptide potently locates mutant proteins to the cell membrane

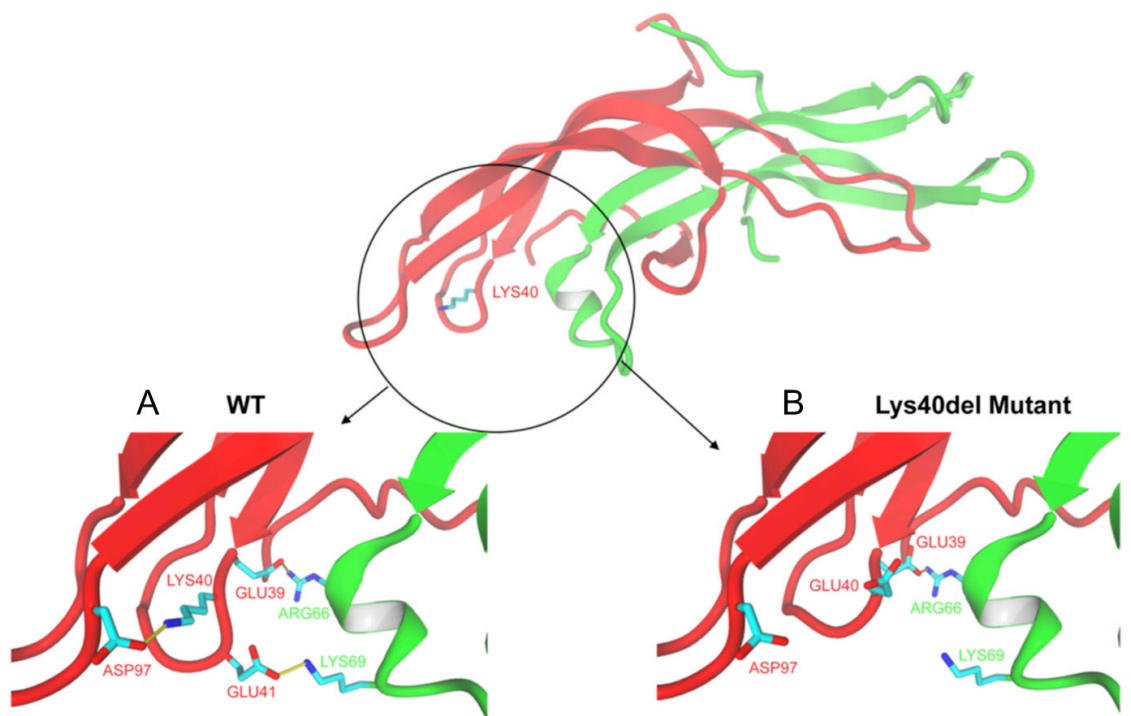

C LHCGR mediated cAMP generation

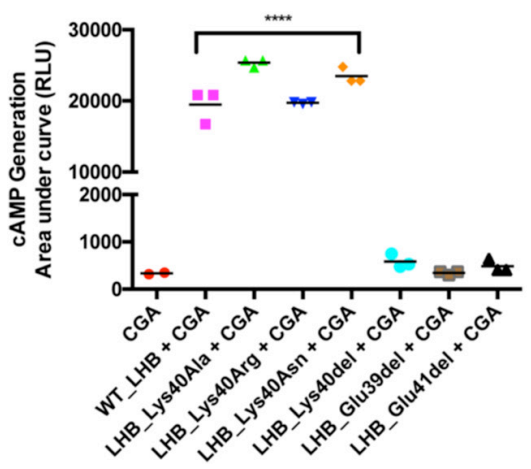

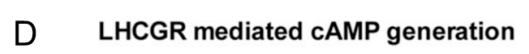

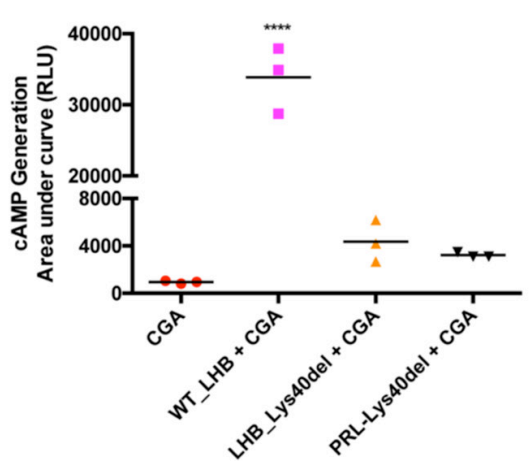

Figure 4

3D modelling and mutagenesis. The WT-LHB and LHB-Lys40del are represented in heterodimerization with CGA (green structure). The position of Lys40 is shown in the WT setting (A) and indicates a potential interaction with Asp97, influencing the loop shape. Absence of Lys 40 results in conformational changes in the loop (B). Adjacent glutamic acids (39 and 41) are show in the WT (A) mutant LHB (B) settings. (C) Mutagenesis of Lys40 to alanine (Lys40Ala), arginine (Lys40Arg), or asparagine (Lys40Asn) and further heterodimerization with CGA and activation of the LHCGR shows that Lys 40 can be replaced by those amino acids without affecting the function of the LHB. Deletions of the glutamic acids before (Glu39del) or after (Glu41del) the Lys40, however, result in the functional inactivation of the LHB. (D) A Lys40del mutant where the signal peptide was swapped for that of prolactin (PRL) also resulted in minimal activation of the LHCGR. The statistical difference ( $P$ value $<0.0001$ ) is highlighted among samples as compared with LHB-Lys40del+CGA. http://joe.endocrinology-journals.org
DOI: $10.1530 /$ JOE-16-0384
(๑) 2016 Society for Endocrinology Printed in Great Britain 
A

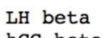

hCG beta

FSH beta

TSH beta

LH beta

hCG_beta

FSH beta

TSHbeta

LH beta

hCG_beta

FSH beta

TSH beta
MEMLQGLLLLLLLSMGGAWASREPLRPWCHPINAILAVEKEGCPVCITVNTTICAGYCPT 60 MEMFOGLLLLLLLSMGGTWASKEPLRPRCRPINATLAVEKEGCPVCITVNTTICAGYCPT 60 MKTLOFF-FLFCCWKAICCN---SCEITNITIAIEKEECRFCISINTTWCAGYCYT 52 MTALFLMSMLFGLTCGOAMS-------FCIPTEYTMHIERRECAYCLTINTTICAGYCMT 53

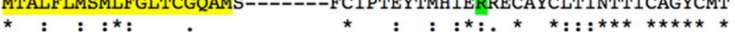

MMRVLQA--VLPPLPQVVCTYRDVRFESIRLPGCPRGVDPVVSFPVALSCRCGPCRRSTS 118 MTRVLQG--VLPALPQVVCNYRDVRFESIRLPGCPRGVNPVVSYAVALSCQCALCRRSTT 118 RDLVYKD-OPRPKIQXTCTEKELVYETVRVPGCAHHADSLYTYPVATQCHCGKCD RDINGKLFLPKYALSQDVCTYRDFIYRTVEIPGCPLHVAPYFSYPVALSCKCGKCNTDYS 113

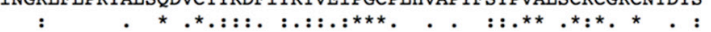

DCGGPKDHPLTCDHPQLSGLLFL-----------------1---1--- 141 DCGGPKDHPLTCDDPRFQDSSSSKAPPPSLPSPSRLPGPSDTPILPQ 165 DCGGPKDHPLTCDDPRFQDSSSSKAPPPSLPSPSRLPGPSDTPILPQ 165

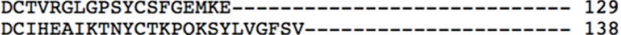

DCIHEAIKTNYCTKPQ

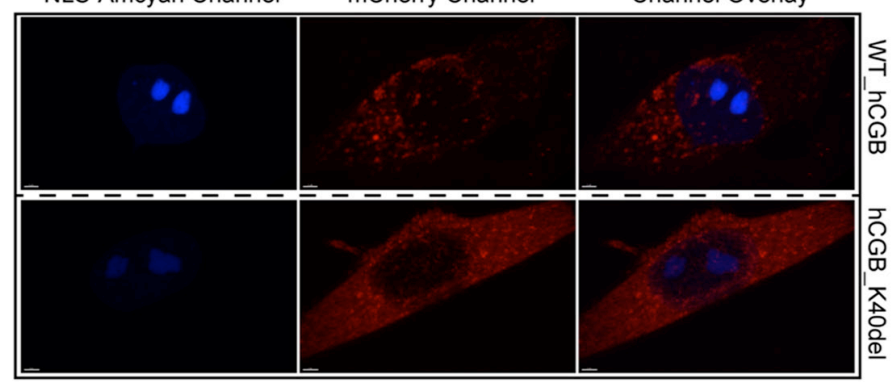

C

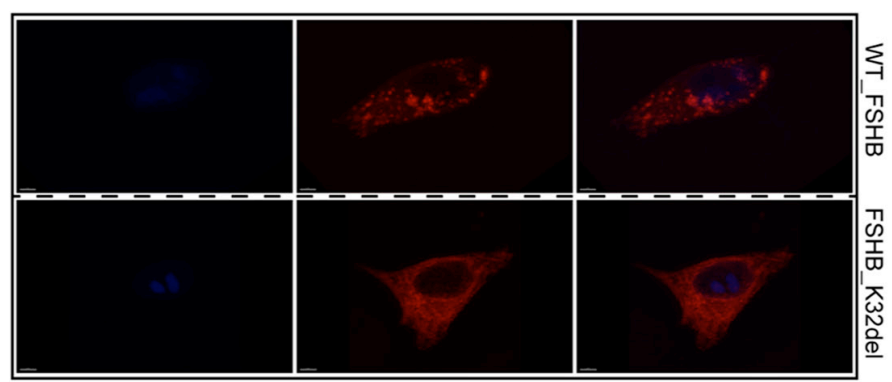

D
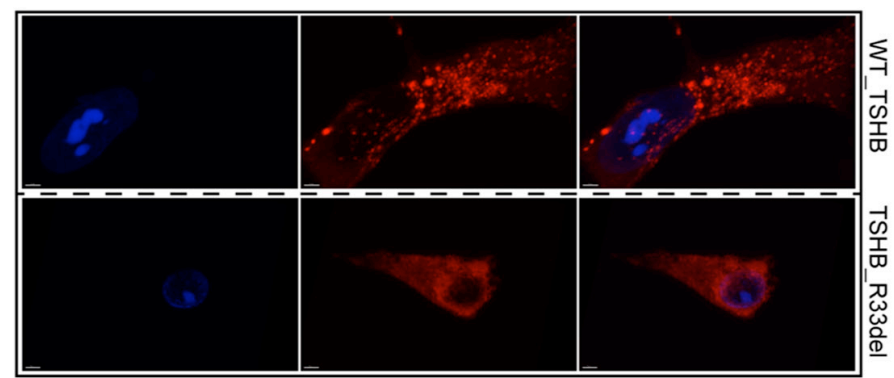

$E$

LHCGR mediated cAMP generation

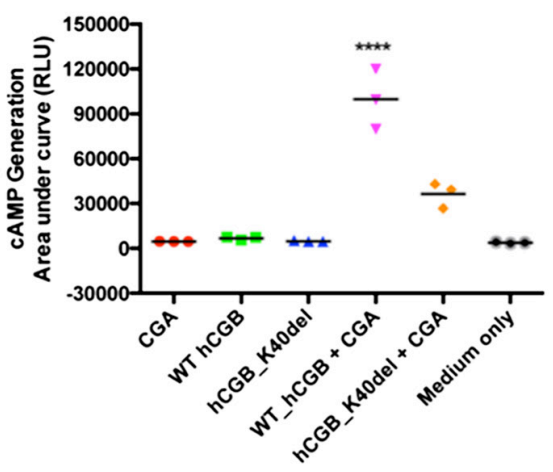

FSHR mediated cAMP generation

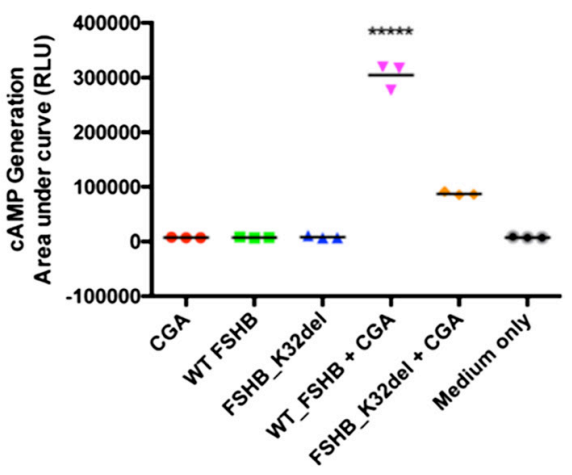

TSHR mediated cAMP generation

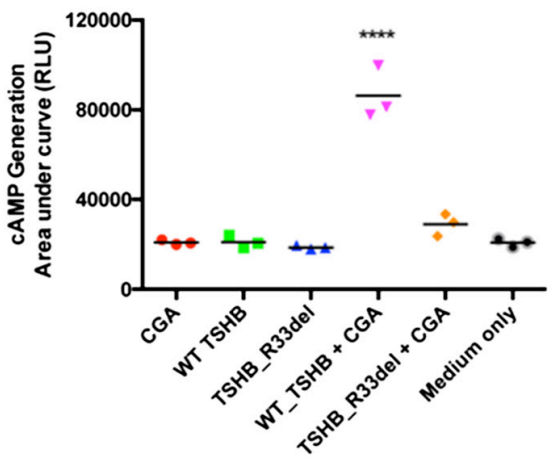

Figure 5

Functional characterization of wild-type and mutant glycoprotein hormone beta subunits. (A) Aligned glycoprotein beta subunits. Signal peptides are highlighted in yellow while the Lys40 (K40) position in LH beta and corresponding positions in other beta subunits are highlighted in green. (B, C, D) Confocal images of HeLa cells expressing WT-hCGB, FSHB and TSHB as fusion proteins with mCherry shows their localization in secretion vesicles. However, the mutant subunits (hCGB-Lys40del, FSHB-Lys32del and TSHB-Arg33del) fused with mCherry, shows an even intracellular localization and markedly reduced localization in secretory vesicles. (E) The lack of localization of hCGB-Lys40del, FSHB-Lys32del and TSHB-Arg33del mutants in secretion vesicles is supported by their reduced ability to stimulate their respective receptors and thus decreased CAMP production as compared with WT-hCGB, WT-FSHB and WT-TSHB, respectively. A statistically significant difference ( $P$ value $<0.0001)$ was found in CAMP production between WT + CGA and mutant + CGA for all glycoprotein hormone beta subunits (hCGB, FSHB and TSHB).

(Rivero-Müller et al. 2010). The intracellular localization of PRL-Lys40del was similar to the LHB-Lys40del (data not shown), and had a similar lack of secretion or LHCGR activation (Fig. 4D).
Given the impact of the Lys40 deletion on LH, we assessed whether analogous amino acids of the other heterodimeric glycoprotein hormone beta-subunits were similarly important for their secretion and function.

Published by Bioscientifica Ltd. 
We aligned the amino acid sequences of human CGB, FSHB, TSHB and LHB with Clustal OMEGA program and the respective positions were deleted to generate hCGB_K40del, FSHB_K32del and TSHB_R33del (Fig. 5A) (Li et al. 2015). Across all human glycoprotein hormones, both cellular localization and cAMP generation were impaired to a very similar extent to that seen with LHB-Lys40del. Compared with WT hormones, these mutant proteins did not localize correctly to secretory vesicles and showed diffuse intracellular distribution (Fig. 5B, C, D). Furthermore, the mutant forms of hCG, FSH and TSH also had greatly decreased secretion and consequently demonstrated reduced activation of their respective receptors as compared with WT hormones, similar to the situation demonstrated with LHB-Lys40del (Fig. 5E).

\section{Discussion}

We report the clinical and molecular characterization of two male patients with delayed puberty due to a homozygous inactivating mutation of the $L H B$ gene in a consanguineous family. The mutation was a deletion of codon 40 of the LHB DNA sequence, which encodes lysine at position 20 of the mature protein (the signal peptide of LH is 20 amino acids). This deletion induces a conformational change in the LH beta-subunit that does not affect its coupling with CGA but prevents correct trafficking of the dimeric hormone molecule through the endoplasmic reticulum, leading to nearly absent secretion. The LHB-Lys40del mutant is secreted $>230$ fold less than WT-LHB, leading to decreased circulating LH that caused hypogonadism in the affected patients. The mutated LHB is, however, capable of dimerizing with CGA and activating the LHCGR.

Male sexual differentiation, development and reproductive function are strictly related to androgen secretion and action. Secretion of androgens depends on the functionality of the hCG-LH-LHCGR axis. In humans, placental hCG binds to the LHCGR, stimulating foetal Leydig cells to secrete testosterone during foetal development, thus ensuring stabilization of the Wolffian ducts and their differentiation into the internal male genital tract (Themmen et al. 2000). Following the conversion of testosterone to dihydrotestosterone the latter induces the development of the prostate and external genital organs. Hence, males with inactivating LHCGR mutations have more severe phenotype at birth (e.g. pseudohermaphroditism) than those with inactivating $L H B$ mutations that are born normal (Latronico \& Arnhold 2013). Postnatally, LH becomes the LHCGR agonist, exerting its effect on adult Leydig cells from puberty onwards. To date, only five other inactivating mutations of the $L H B$ have been described (Weiss et al. 1992, Valdes-Socin et al. 2004, LofranoPorto et al. 2007, Achard et al. 2009, Basciani et al. 2012). Their clinical presentation is similar with pubertal arrest in males (Axelrod et al. 1979, Valdes-Socin et al. 2004, Lofrano-Porto et al. 2007, Achard et al. 2009, Basciani et al . 2012) and secondary amenorrhoea/oligomenorrhoea, anovulation and infertility in females despite normal secondary sexual characteristics (Lofrano-Porto et al. 2007, Achard et al. 2009, Basciani et al. 2012). Due to the more dramatic clinical presentation, $L H B$ mutations were initially found only in males; the female cases described are sisters of male index patients. Treatment of the two patients described here with hCG-normalized testosterone secretion, thereby enabling the acquisition of secondary sexual characteristics and improvement in sperm production.

Previous instances of LH inactivation were due to homozygous mutations that prevented binding to LHCGR (Axelrod et al. 1979, Weiss et al. 1992) or the formation of heterodimers (Valdes-Socin et al. 2004), deleted important cysteine residues (H30-I32del) (Achard et al. 2009), caused abnormal splicing of the LH beta-subunit (Lofrano-Porto et al. 2007) or presented with a compound heterozygous mutation of the signal peptide (L9-L12del) in combination with a mutation at an intronic splice donor site on the other allele (Basciani et al. 2012).

The genetic mechanism impairing LH action in this family differs from the others described previously. We showed that the Lys40del did not impair the expression of $L H B$, prevent heterodimerization or alter the ratio of LHB monomer to dimer. Furthermore, the mutated dimer bound to and activated its receptor, LHCGR. Faulty trafficking of the mutated dimeric LH caused absent LH secretion; instead of targeted transport to secretory vesicles, the mutated $\mathrm{LH}$ diffused generally into the cytoplasm. Substituting Lys40 with either a hydrophilic (arginine), a hydrophobic (alanine) or a neutral residue (asparagine) did not impair LH function. Deleting either of the glutamic acids that flank Lys40 resulted in an identical effect on receptor stimulation to that seen with Lys40del. This indicates that amino acids in this region are crucial for correct intracellular trafficking of LH during its synthesis, and deletions result in intracellular retention, lack of LH secretion and the observed clinical phenotype. The 3D model indicates that Lys40 is situated in a loop

Published by Bioscientifica Ltd. 
between alpha helices and alterations in this loop appear to trigger a change in the peptide 3D structure.

Although the glycoprotein hormone family regulates diverse biological functions, the individual hormones share a significant structural similarity ( $\mathrm{Li}$ \& Ford 1998). The CGA and beta-subunits belong to the cystine-knot superfamily and have a similar tertiary structure with three elongated loops (Cahoreau et al. 2015). The $C$-terminal segment of the beta-subunit stabilizes the heterodimer by wrapping around the alpha-subunit like a seat belt and latching through a disulphide bond with a cysteine residue from loop 1 of the beta-subunit (Lapthorn et al. 1994, Xing et al. 2001, 2004). Lys40 is situated in the beta- 1 loop, close to a cysteine residue that is part of the seat belt latch. However, deletion of Lys40 or its flanking glutamic acid residues appears not to impair the assembly of functional dimeric LH. Intriguingly, analogous mutations of hCG, FSH and TSH beta-subunits had a very similar effect to that seen in $\mathrm{LH}$, with marked intracellular retention, minimal secretion and decreased receptor stimulation. This general effect of deletions in this discrete region across the glycoprotein betasubunits suggests impairment of a common step that precedes trafficking of hormones to secretory vesicles. This is most likely due to protein misfolding after the protein is undocked from the endoplasmic reticulum and beforeitenters the Golgiapparatus. The cytosolic diffusion of mutated protein seen on confocal microscopy for all the glycoprotein hormones suggests that misfolded protein has been re-exported out of the endoplasmic reticulum. While we would speculate that the misfolded glycoprotein hormone would undergo ubiquitination and proteasomal destruction, their continued presence throughout the cytoplasm argues against rapid degradation (Smith et al. 2011).

In conclusion, the clinical phenotype of hypogonadism in two brothers with a homozygous $L H B$ mutation causing a single amino acid deletion reveals an important region for normal secretion of all four human glycoprotein hormones. These discrete changes led to intracellular retention of functional dimeric hormones that can have important clinical consequences. Additionally, we have developed a new system to test mutations in glycoprotein hormones that allows for easy visualization of expression and localization.

\section{Supplementary data}

This is linked to the online version of the paper at http://dx.doi.org/10.1530/ JOE-16-0384.

\section{Declaration of interest}

The authors declare that there is no conflict of interest that could be perceived as prejudicing the impartiality of the research reported.

\section{Funding}

The study was supported by grants from the Academy of Finland, Turku Doctoral Programme of Biomedical Sciences (TuBS), Turku Doctoral Programme of Molecular Medicine (TuDMM), the Sigrid Jusélius Foundation, the Polish National Science Center UMO-2015/17/B/NZ1/01777, and the Fonds d'Investissement pour la Recherche (FIRS) of the Centre Hospitalier Universitaire de Liège, Belgium.

\section{Materials and correspondence}

Any request for materials used in the manuscript can be forwarded to A T, A R-M, A F D or A B.

\section{Author contribution statement}

A F D, A R-M and I P designed the experiments. I P, A H, J-J M, F P. A B and A F D designed, performed or oversaw clinical and genetic studies. A R-M and A T participated in design and construction of plasmid vectors and conducted the functional tests. A T participated in visualization of betasubunits by confocal microscopy. A Th conducted pathological studies of testicular biopsy. $\mathrm{M} \mathrm{K}$ generated the diffusion data. $\mathrm{KJ}$ performed the 3D modelling. I P, A R-M, A T and A F D wrote the manuscript. I H and $A B$ coordinated the research. All authors read and approved the final manuscript. I H, A B and A F D contributed equally to the conceptualization of this work.

\section{Acknowledgements}

The authors acknowledge Dr Roberto Salvi and Dr Maria Cristina Burlacu for contributions to the genetic and clinical studies.

\section{References}

Achard C, Courtillot C, Lahuna O, Méduri G, Soufir JC, Lière P, Bachelot A, Benyounes H, Schumacher M, Kuttenn F, et al. 2009 Normal spermatogenesis in a man with mutant luteinizing hormone. New England Journal of Medicine 361 1856-1863. (doi:10.1056/ NEJMoa0805792)

Ahtiainen P, Sharp V, Rulli SB, Rivero-Müller A, Mamaeva V, Röyttä M \& Huhtaniemi I 2010 Enhanced LH action in transgenic female mice expressing hCGbeta-subunit induces pituitary prolactinomas; the role of high progesterone levels. Endocrine-Related Cancer 17 611-621. (doi:10.1677/ERC-10-0016)

Axelrod L, Neer RM \& Kliman B 1979 Hypogonadism in a male with immunologically active, biologically inactive luteinizing hormone: an exception to a venerable rule. Journal of Clinical Endocrinology and Metabolism 48 279-287. (doi:10.1210/jcem-48-2-279)

Basciani S, Watanabe M, Mariani S, Passeri M, Persichetti A, Fiore D, Scotto d'Abusco A, Caprio M, Lenzi A, Fabbri A, et al. 2012 Hypogonadism in a patient with two novel mutations of the luteinizing hormone beta-subunit gene expressed in a compound heterozygous form. Journal of Clinical Endocrinology and Metabolism 97 3031-3038. (doi:10.1210/jc.2012-1986)

Cahoreau C, Klett D \& Combarnous Y 2015 Structure-function relationships of glycoprotein hormones and their subunits' ancestors. Frontiers in Endocrinology 6 26. (doi:10.3389/fendo.2015.00026)

Cardarelli F, Tosti L, Serresi M, Beltram F \& Bizzarri R 2012 Fluorescent recovery after photobleaching (FRAP) analysis of nuclear export rates 
identifies intrinsic features of nucleocytoplasmic transport. Journal of Biological Chemistry 287 5554-5561. (doi:10.1074/jbc.M111.304899)

Lapthorn AJ, Harris DC, Littlejohn A, Lustbader JW, Canfield RE, Machin KJ, Morgan FJ \& Isaacs NW 1994 Crystal structure of human chorionic gonadotropin. Nature 369 455-461. (doi:10.1038/369455a0)

Latronico AC \& Arnhold IJ 2013 Gonadotropin resistance. Endocrine Development 24 25-32. (doi:10.1159/000342496)

Li M \& Ford J 1998 A comprehensive evolutionary analysis based on nucleotide and amino acid sequences of the alpha- and beta-subunits of glycoprotein hormone gene family. Journal of Endocrinology 156 529-542. (doi:10.1677/joe.0.1560529)

Lofrano-Porto A, Barra GB, Giacomini LA, Nascimento PP, Latronico AC, Casulari LA \& da Rocha Neves Fde A 2007 Luteinizing hormone beta mutation and hypogonadism in men and women. New England Journal of Medicine 357 897-904. (doi:10.1056/NEJMoa071999)

Rivero-Müller A, Chou YY, Ji I, Lajic S, Hanyaloglu AC, Jonas K, Rahman N, Ji TH \& Huhtaniemi I 2010 Rescue of defective G proteincoupled receptor function in vivo by intermolecular cooperation. PNAS 107 2319-2324.

Smith MH, Ploegh HL \& Weissman JS 2011 Road to ruin: targeting proteins for degradation in the endoplasmic reticulum. Science $\mathbf{3 3 4}$ 1086-1090. (doi:10.1126/science.1209235)

Themmen APN \& Huhtaniemi IT 2000 Mutations of gonadotropins and gonadotropin receptors: elucidating the physiology and pathophysiology of pituitary-gonadal function. Endocrine Reviews 21 551-583. (doi:10.1210/edrv.21.5.0409)

Trehan A, Kielbus M, Czapinski J, Stepulak A, Huhtaniemi I \& Rivero-Müller A 2016 REPLACR-mutagenesis, a one-step method for site-directed mutagenesis by recombineering. Scientific Reports 6 19121. (doi:10.1038/srep19121)

Trehan A, Rotgers E, Coffey ET, Huhtaniemi I \& Rivero-Muller A 2014 CANDLES, an assay for monitoring GPCR induced cAMP generation in cell cultures. Cell Communication and Signalling 1270. (doi:10.1186/s12964-014-0070-x)

Valdes-Socin H, Salvi R, Daly AF, Gaillard RC, Quatresooz P, Tebeu PM, Pralong FP \& Beckers A 2004 Hypogonadism in a patient with a mutation in the luteinizing hormone beta-subunit gene. New England Journal of Medicine 351 2619-2625. (doi:10.1056/ NEJMoa040326)

Valdes-Socin H, Salvi R, Thiry A, Daly AF, Pralong FP, Gaillard R \& Beckers A 2009 Testicular effects of isolated luteinizing hormone deficiency and reversal by long-term human chorionic gonadotropin treatment. Journal of Clinical Endocrinology and Metabolism 94 3-4. (doi:10.1210/jc.2008-1584)

Weiss J, Axelrod L, Whitcomb RW, Harris PE, Crowley WF \& Jameson JL 1992 Hypogonadism caused by a single amino acid substitution in the beta subunit of luteinizing hormone. New England Journal of Medicine 326 179-183. (doi:10.1056/NEJM199201163260306)

Xing Y, Lin W, Jiang M, Myers RV, Cao D, Bernard MP \& Moyle WR 2001 Alternatively folded choriogonadotropin analogs: implications for hormone folding and biological activity. Journal of Biological Chemistry 276 46953-46960. (doi:10.1074/jbc.M108374200)

Xing Y, Myers RV, Cao D, Lin W, Jiang M, Bernard MP \& Moyle WR 2004 Glycoprotein hormone assembly in the endoplasmic reticulum: III. The seatbelt and its latch site determine the assembly pathway. Journal of Biological Chemistry 279 35449-35457. (doi:10.1074/jbc. M403054200)

Received in final form 9 September 2016

Accepted 21 September 2016

Accepted Preprint published online 21 September 2016
(C) 2016 Society for Endocrinology Printed in Great Britain 